\title{
BOUNDARY INTEGRAL FORMULAE FOR THE RECONSTRUCTION OF ELECTRIC AND ELECTROMAGNETIC INHOMOGENEITIES OF SMALL VOLUME
}

\author{
Habib Ammari ${ }^{1}$, Shari Moskow ${ }^{2}$ and Michael S. Vogelius ${ }^{3}$
}

\begin{abstract}
In this paper we discuss the approximate reconstruction of inhomogeneities of small volume. The data used for the reconstruction consist of boundary integrals of the (observed) electromagnetic fields. The numerical algorithms discussed are based on highly accurate asymptotic formulae for the electromagnetic fields in the presence of small volume inhomogeneities.
\end{abstract}

Mathematics Subject Classification. 35J25, 35R30, 65R99.

Received May 1, 2001.

\section{INTRODUCTION}

The problem of determining interior information about a medium from boundary field measurements is one that is not in general well posed. If, however, in advance we have additional structural information about the medium, then we may be able to determine specific features with "higher resolution". As an example consider a bounded domain consisting of a homogeneously conducting background medium with volumetrically small inhomogeneities of conductivity different from the background. The geometry of each of the inhomogeneities may take the form of

$$
\epsilon B+z,
$$

where $B$ is some fixed bounded domain containing the origin, or,

$$
\left\{x+\eta h(x) n(x): x \in \sigma_{0}, \eta \in(-\epsilon, \epsilon)\right\},
$$

where $\sigma_{0}$ is a simple curve, $n(x)$ is a unit vectorfield orthogonal to $\sigma_{0}$, and $h(x)$ is some smooth positive function. That is, we assume the inhomogeneities are either small domains of diameter $\approx \epsilon$, or they are thin strips of thickness $\approx \epsilon$ (this latter case only pertains to two dimensions). When in the shape of strips, the inhomogeneities are required to have bounded, nonzero conductivity, in order that the limiting $(\epsilon \rightarrow 0)$ voltage potential coincide with that of the pure background medium.

In both of the settings described above, asymptotic formulae have been derived for the voltage potential as $\epsilon \rightarrow 0$. These formulae express the $\epsilon$-dependent voltage potential in terms of the background potential, a certain

Keywords and phrases: Electromagnetic imaging, small inhomogeneities, numerical reconstruction algorithms.

1 CMAP, École Polytechnique, 91128 Palaiseau, France.

2 Department of Mathematics, University of Florida, Gainesville, FL 32611, USA.

3 Department of Mathematics, Rutgers University, New Brunswick, NJ 08903, USA; e-mail: vogelius@hilbert.rutgers.edu 
Neumann-function, and the relevant polarization tensors [5,8,11]. In this paper we will use these formulae to develop an efficient algorithm to determine the location and/or shape of the inhomogeneities.

To be quite precise, let us assume we are given some finite set of small inhomogeneities $\omega_{\epsilon}$

$$
\omega_{\epsilon}=\bigcup_{j=1}^{m} \omega_{\epsilon}^{j}
$$

where, either all the inhomogeneities are of the form

$$
\omega_{\epsilon}^{j}=\epsilon B_{j}+z_{j},
$$

for some smooth, bounded, star-shaped domains, $B_{j}$, or they are all of the form

$$
\omega_{\epsilon}^{j}=\left\{x+\eta h^{(j)}(x) n(x): x \in \sigma_{0}^{j}, \eta \in(-\epsilon, \epsilon)\right\},
$$

for some simple curves, $\sigma_{0}^{j}$. Here $n(x)$ is a unit normal vectorfield to $\sigma_{0}^{j}$, and $h^{(j)}$ is some positive smooth function. The potential $u_{\epsilon}$ satisfies

$$
\begin{aligned}
\nabla \cdot \gamma_{\epsilon} \nabla u_{\epsilon} & =0 \quad \text { in } \Omega \\
\gamma_{\epsilon} \frac{\partial u_{\epsilon}}{\partial \nu}=g & \text { on } \partial \Omega
\end{aligned}
$$

with conductivity

$$
\gamma_{\epsilon}=\left\{\begin{array}{cc}
\gamma_{0} & x \in \Omega \backslash \bar{\omega}_{\epsilon} \\
\gamma_{j} & x \in \omega_{\epsilon}^{j}
\end{array}\right.
$$

We know that as $\epsilon$ approaches zero, the potential $u_{\epsilon}$ converges to the background potential, $u$, which satisfies

$$
\begin{aligned}
\Delta u=0 & \text { in } \quad \Omega, \\
\gamma_{0} \frac{\partial u}{\partial \nu}=g & \text { on } \quad \partial \Omega .
\end{aligned}
$$

In the case of $m$ star-shaped imperfections of type $\epsilon B_{j}+z_{j}$ (of either finite or extreme conductivity) that are well separated from each other and from the boundary, it has been shown in $[8,11]$ that the following asymptotic formula holds:

$$
u_{\epsilon}(y)=u(y)+\epsilon^{n} \sum_{j=1}^{m}\left(\frac{\gamma_{0}}{\gamma_{j}}-1\right) \nabla_{x} N\left(z_{j}, y\right) \cdot M^{(j)} \nabla u\left(z_{j}\right)+o\left(\epsilon^{n}\right)
$$

$N(x, y)$ is the standard Neumann function

$$
\begin{aligned}
\Delta_{x} N(x, y) & =-\delta_{y} \quad \text { in } \quad \Omega \\
\frac{\partial N}{\partial \nu_{x}}(x, y) & =-\frac{1}{|\partial \Omega|} \quad \text { on } \quad \partial \Omega,
\end{aligned}
$$

and each $M^{(j)}$ is a $n \times n$, symmetric, positive definite matrix (called the polarization tensor). $M^{(j)}$ is given by

$$
M_{k l}^{(j)}=\left|B_{j}\right| \delta_{k l}-\int_{\partial B_{j}} y_{k} \frac{\partial \phi_{l}^{+}}{\partial \nu} \mathrm{d} \sigma_{y}
$$


where, for $0<c_{j}=\frac{\gamma_{0}}{\gamma_{j}}<\infty$ and $1 \leq l \leq n, \phi_{l}(y)$ is the unique solution to

$$
\begin{aligned}
\Delta \phi_{l} & =0 \quad \text { in } B_{j}, \\
\Delta \phi_{l} & =0 \text { in } \mathbb{R}^{n} \backslash \bar{B}_{j}, \\
c_{j} \frac{\partial \phi_{l}^{+}}{\partial \nu}-\frac{\partial \phi_{l}^{-}}{\partial \nu} & =\left(c_{j}-1\right) \nu_{l} \quad \text { on } \partial B_{j},
\end{aligned}
$$

with $\phi_{l}$ continuous across $\partial B_{j}$, and

$$
\lim _{|y| \rightarrow \infty} \phi_{l}(y)=0
$$

Note that the polarization tensor depends on the conductivity aspect ratio and the size and the shape of the inhomogeneity. Also note that the above asymptotic formula for $u_{\epsilon}$ holds in $\mathbb{R}^{n}$ for any $n \geq 2$ (later we shall specialize to two dimensions). It is sometimes convenient to emphasize the dependence of $M^{(j)}$ on the conductivity aspect ratio, $c_{j}=\gamma_{0} / \gamma_{j}$, in which case we write $M^{(j)}\left(c_{j}\right)$. The tensor $M^{(j)}$, introduced here, is the same as that introduced in [8], and is exactly $c_{j}^{-1}$ times that introduced in [16].

There are very similar formulae for the (rescaled) polarization tensor in the case $\gamma_{j}=0$ or $\gamma_{j}=\infty$, see [8] and [11]. The remainder, $o\left(\epsilon^{n}\right)$ in (1.4) signifies a term which goes to zero faster than $\epsilon^{n}$ uniformly in $y$, for $y$ bounded away from the inhomogeneities. (It has been shown rigorously that this term is $O\left(\epsilon^{n+1 / 2}\right)$, but numerical computations seem to suggest it goes to zero faster than this when the $B_{j}$ are smooth.)

Remark 1. If (for fixed inhomogeneity $B_{j}$ ) we define $\varphi_{l}=-\phi_{l}+y_{l}$, then $\varphi_{l}$ satisfies

$$
\begin{aligned}
& \nabla_{y} \cdot \gamma(y) \nabla_{y} \varphi_{l}=0 \quad \text { in } \mathbb{R}^{n} \\
& \varphi_{l}-y_{l} \rightarrow 0 \quad \text { as }|y| \rightarrow \infty
\end{aligned}
$$

Here $\gamma(y)$ is given by

$$
\gamma(y)=\left\{\begin{array}{cc}
\gamma_{0} & y \in \mathbb{R}^{n} \backslash \bar{B}_{j} \\
\gamma_{j} & x \in B_{j}
\end{array}\right.
$$

A simple calculation now shows that

$$
M_{k l}^{(j)}=c_{j}^{-1} \int_{B_{j}} \frac{\partial}{\partial y_{k}} \varphi_{l}(y) \mathrm{d} y
$$

For $n=2$ there is a very special relationship between the polarization tensor corresponding to aspect ratio $c$ and that corresponding to aspect ratio $c^{-1}$. Based on the formula just above, and convex duality, we get that

$$
M^{(j)}\left(c_{j}^{-1}\right)=c_{j} R M^{(j)}\left(c_{j}\right) R^{T}
$$

where $R$ denotes the matrix corresponding to a counterclockwise rotation by $\pi / 2$, i.e., the matrix given by $R_{11}=R_{22}=0, R_{12}=-R_{21}=-1$.

When the $m$ conductivity imperfections $\omega_{\epsilon}^{j}$ all take the form of the thin strips described above, and when these strips are well separated from each other and from the boundary, a similar asymptotic formula has been formally derived in $[5]$ :

$$
u_{\epsilon}(y)=u(y)-\epsilon \sum_{j=1}^{m} \int_{\sigma_{0}^{j}} \nabla_{x} N(x, y) \cdot A^{(j)}(x) \nabla u(x) h^{(j)}(x) \mathrm{d} s_{x}+o(\epsilon) .
$$


The matrix $A^{(j)}(x)$ is similar to the polarization tensor $M^{(j)}$. If $\tau(x)$ and $n(x)$ are the unit tangential and normal fields to $\sigma_{0}^{j}$, then $A^{(j)}(x)$ is the symmetric matrix with eigenvectors $\tau(x)$ and $n(x)$, with respective eigenvalues $2\left(c_{j}^{-1}-1\right)$ and $2\left(1-c_{j}\right)$. Although this formula has not been proven rigorously, the computational evidence in [5] attests to its accuracy. We also note that this formula only pertains to two dimensions.

Our first goal is to apply these asymptotic formulae for the purpose of identifying the location and certain properties of the shapes of the inhomogeneities. In contrast to a (straightforward) boundary least squares fit to "the measured data", we develop a method based on appropriate averaging, using particular "background" solutions as weights. The order $\epsilon^{n}$ term in the representation formula (1.4) is physically that of a voltage potential corresponding to $m$ appropriately polarized dipoles located at the points $z_{j}, 1 \leq j \leq m$. Similarly the order $\epsilon$ term in (1.10) is an "average" of voltage potentials corresponding to appropriately polarized dipoles distributed along the curve $\sigma_{0}$. Results that address the theoretical feasibility of recovering the location of dipoles from boundary measurements are found various places in the literature; the reader may for example consult [14] and [12]. The special formulae found in [12] Section 3 for the recovery of a single inhomogeneity are very related to our brief discussion in Section 3 of the present paper.

Our second goal is to formally show that a similar "small volume" perturbation approach may be applied to the full Maxwell equations with inhomogeneities of different electric permittivity or magnetic permeability (or both). The formal arguments we present provide excellent insight when it comes to the inner workings of this "small volume" perturbation approach - an insight which may hopefully be used to adapt the approach for use in other contexts (for instance in the context of spectral data).

\section{Integration AGAinst SPECIAL TEST FUNCTIONS}

The general approach we take to reconstruct the locations and certain properties of the shapes of the inhomogeneities is to form the integral of "the measured boundary data" against harmonic test functions. In this section we use the formulae (1.4) and (1.10) to derive alternate expressions for these boundary integrals. These expressions are rather explicit in terms of the inhomogeneities and their locations. Suppose $u_{\epsilon}$ is the solution to (1.1) and $w$ is some known harmonic function. We look for an expression for the "averaged measurement"

$$
\Gamma=\int_{\partial \Omega} u_{\epsilon} \gamma_{0} \frac{\partial w}{\partial \nu} \mathrm{d} \sigma-\int_{\partial \Omega} \gamma_{\epsilon} \frac{\partial u_{\epsilon}}{\partial \nu} w \mathrm{~d} \sigma
$$

Integrals like $\Gamma$ have already been used in several contexts to determine "interior" information from boundary data, most notably to determine the location of plane cracks, see for instance [2,3] or [4]. If, in (2.11), we replace $u_{\epsilon}$ with the right hand side of (1.4), and $\gamma_{\epsilon} \frac{\partial u_{\epsilon}}{\partial \nu}$ with $g$, then we obtain

$$
\Gamma=\int_{\partial \Omega} u \gamma_{0} \frac{\partial w}{\partial \nu} \mathrm{d} \sigma+\epsilon^{n} \sum_{j=1}^{m}\left(\frac{\gamma_{0}}{\gamma_{j}}-1\right) \int_{\partial \Omega} \nabla_{x} N\left(z_{j}, y\right) \cdot M^{(j)} \nabla u\left(z_{j}\right) \gamma_{0} \frac{\partial w}{\partial \nu}(y) \mathrm{d} \sigma_{y}-\int_{\partial \Omega} g w \mathrm{~d} \sigma+o\left(\epsilon^{n}\right) .
$$

The first and third terms cancel due to the harmonicity of $u$ and $w$ (and the fact that $\gamma_{0} \frac{\partial u}{\partial \nu}=g$ ) and we are left with

$$
\Gamma=\epsilon^{n} \sum_{j=1}^{m}\left(\frac{\gamma_{0}}{\gamma_{j}}-1\right) M^{(j)} \nabla u\left(z_{j}\right) \cdot \nabla_{x} \int_{\partial \Omega} N\left(z_{j}, y\right) \gamma_{0} \frac{\partial w}{\partial \nu}(y) \mathrm{d} \sigma_{y}+o\left(\epsilon^{n}\right) .
$$

By integration by parts and use of the equation for $N$ we have

$$
\begin{gathered}
\int_{\partial \Omega} N(x, y) \frac{\partial w}{\partial \nu} \mathrm{d} \sigma_{y}=w(x)-\frac{1}{|\partial \Omega|} \int_{\partial \Omega} w \mathrm{~d} \sigma, \quad \text { or } \\
\nabla_{x} \int_{\partial \Omega} N(x, y) \gamma_{0} \frac{\partial w}{\partial \nu} \mathrm{d} \sigma_{y}=\gamma_{0} \nabla w(x) .
\end{gathered}
$$


We have thus established the following theorem:

Theorem 1. Let $u_{\epsilon}$ be the solution to (1.1) with inhomogeneities of type $\epsilon B_{j}+z_{j}$, with polarization tensors $M^{(j)}$. Let $u$ be the background potential, satisfying (1.3). If $w$ is any harmonic function, then the measurement $\Gamma$, defined by (2.11), has the expansion

$$
\Gamma=\epsilon^{n} \sum_{i=j}^{m} \gamma_{0}\left(\frac{\gamma_{0}}{\gamma_{j}}-1\right) M^{(j)} \nabla u\left(z_{j}\right) \cdot \nabla w\left(z_{j}\right)+o\left(\epsilon^{n}\right)
$$

The same argument can be applied to the case of strips. By replacing $u_{\epsilon}$ with the expansion (1.10) we obtain the approximation

$$
\Gamma=-\epsilon \sum_{j=1}^{m} \int_{\partial \Omega} \int_{\sigma_{0}^{j}} \nabla_{x} N(x, y) A^{(j)}(x) \nabla u(x) h^{(j)}(x) \gamma_{0} \frac{\partial w}{\partial \nu}(y) \mathrm{d} s_{x} \mathrm{~d} \sigma_{y}+o(\epsilon)
$$

which, by interchange of the order of integration, yields

$$
\Gamma=-\epsilon \sum_{j=1}^{m} \int_{\sigma_{0}^{j}} A^{(j)}(x) \nabla u(x) \cdot \nabla_{x} \int_{\partial \Omega} N(x, y) \gamma_{0} \frac{\partial w}{\partial \nu}(y) \mathrm{d} \sigma_{y} h^{(j)}(x) \mathrm{d} s_{x}+o(\epsilon) .
$$

Now, due to (2.12), we obtain

$$
\Gamma=-\epsilon \sum_{j=1}^{m} \gamma_{0} \int_{\sigma_{0}^{j}} \nabla w(x) \cdot A^{(j)}(x) \nabla u(x) h^{(j)}(x) \mathrm{d} s_{x}+o(\epsilon) .
$$

We note that this last expansion has not been completely rigorously verified (due to the fact that the derivation of (1.10) is only formal).

To summarize: we have at this point at our disposal two asymptotic formulae with which to reconstruct the inhomogeneities; if the inhomogeneities are all of small diameter, then

$$
\int_{\partial \Omega} u_{\epsilon} \gamma_{0} \frac{\partial w}{\partial \nu} \mathrm{d} \sigma-\int_{\partial \Omega} \gamma_{\epsilon} \frac{\partial u_{\epsilon}}{\partial \nu} w \mathrm{~d} \sigma \approx \epsilon^{n} \sum_{j=1}^{m} \gamma_{0}\left(\frac{\gamma_{0}}{\gamma_{j}}-1\right) M^{(j)} \nabla u\left(z_{j}\right) \cdot \nabla w\left(z_{j}\right)
$$

and if the inhomogeneities are all thin strips (in two dimensions) then

$$
\int_{\partial \Omega} u_{\epsilon} \gamma_{0} \frac{\partial w}{\partial \nu} \mathrm{d} \sigma-\int_{\partial \Omega} \gamma_{\epsilon} \frac{\partial u_{\epsilon}}{\partial \nu} w \mathrm{~d} \sigma \approx-\epsilon \sum_{j=1}^{m} \gamma_{0} \int_{\sigma_{0}^{j}} \nabla w(x) \cdot A^{(j)}(x) \nabla u(x) h^{(j)}(x) \mathrm{d} s_{x}
$$

In both cases $w$ is an arbitrary harmonic function.

Remark 2. Let us restrict attention to two dimensions. If we were to consider the simultaneous presence of thin inhomogeneities of thickness $\epsilon^{2}$ and diametrically small inhomogeneities of diameter $\epsilon$ (i.e., differently shaped inhomogeneities of same magnitude area) then a direct, additive combination of the formulae (2.13) and (2.14) gives the leading order perturbation of the "averaged data". Perhaps more interesting, one might calculate a higher order version of (2.14) (including terms of order $\epsilon^{2}$ ) and by combination with (2.13) now obtain an asymptotic perturbation for the "averaged data" in the simultaneous (well separated) presence of thin strips and diametrically small inhomogeneities, with thickness and diameter of same magnitude, $\epsilon$. 
Remark 3. If $\gamma_{\epsilon}$ is a small amplitude perturbation (an $L^{\infty}$-small perturbation) of the constant $\gamma_{0}$, i.e., if $\gamma_{\epsilon}(x)=\gamma_{0}+\epsilon \gamma(x)$, with $\gamma \in L^{\infty}(\Omega)$, then it is not difficult to show that

$$
\int_{\partial \Omega} u_{\epsilon} \gamma_{0} \frac{\partial w}{\partial \nu} \mathrm{d} \sigma-\int_{\partial \Omega} \gamma_{\epsilon} \frac{\partial u_{\epsilon}}{\partial \nu} w \mathrm{~d} \sigma=-\epsilon \int_{\Omega} \gamma(x) \nabla u_{\epsilon} \cdot \nabla w \mathrm{~d} x \approx-\epsilon \int_{\Omega} \gamma(x) \nabla u \cdot \nabla w \mathrm{~d} x,
$$

for any harmonic function $w$. This approximation was for example used by Calderon [7]. The formulae (2.13) and (2.14) may be viewed as the analogues of (2.15) for special "low volume fraction perturbations", that is for special types of $L^{1}$-small perturbations.

\section{Detecting one inhomogeneity}

In this section we look at the simple case of only one inhomogeneity and show how formula (2.13) can be used to approximate its location and its polarization tensor. Suppose the inhomogeneity is centered at the point $p=\left(p_{1}, p_{2}\right)$, and has polarization tensor $M$. For simplicity we here denote the "rescaled" polarization tensor $\gamma_{0}\left(\frac{\gamma_{0}}{\gamma_{1}}-1\right) M$ by $M$, and we consider only the case $n=2$. Then, for any harmonic $w$

$$
\int_{\partial \Omega} u_{\epsilon} \gamma_{0} \frac{\partial w}{\partial \nu} \mathrm{d} \sigma-\int_{\partial \Omega} \gamma_{\epsilon} \frac{\partial u_{\epsilon}}{\partial \nu} w \mathrm{~d} \sigma \approx \epsilon^{2} M \nabla u(p) \cdot \nabla w(p) .
$$

Let us neglect the asymptotically small remainder term, and apply the currents $\left(g_{i}=\gamma_{0} \nu_{i}\right)$ corresponding to background potentials

$$
u^{(i)}=x_{i}, \quad i=1,2 .
$$

First, we use the two test functions

$$
w^{(1)}=x_{1} \text {, and } w^{(2)}=x_{2} .
$$

Define the "measurement" $\Gamma_{i j}$ by

$$
\Gamma_{i j}=\int_{\partial \Omega} u_{\epsilon}^{(i)} \gamma_{0} \frac{\partial w^{(j)}}{\partial \nu} \mathrm{d} \sigma-\int_{\partial \Omega} \gamma_{\epsilon} \frac{\partial u_{\epsilon}^{(i)}}{\partial \nu} w^{(j)} \mathrm{d} \sigma
$$

From (3.16) we immediately get an approximation to the terms of the tensor $\epsilon^{2} M$. In fact,

$$
\epsilon^{2} M_{i j}=\Gamma_{i j}
$$

Now that we have "recovered" $\epsilon^{2} M$, we may use the same two currents, $\gamma_{0} \nu_{1}$ and $\gamma_{0} \nu_{2}$, to determine the location of the inhomogeneity. By inserting the test function

$$
w^{(3)}=x_{1} x_{2}
$$

into (3.16), we obtain the linear system

$$
\begin{aligned}
& \Gamma_{13}=\epsilon^{2}\left(M_{11} p_{2}+M_{21} p_{1}\right), \\
& \Gamma_{23}=\epsilon^{2}\left(M_{12} p_{2}+M_{22} p_{1}\right),
\end{aligned}
$$

which, since we already know $\epsilon^{2} M$, is solvable for $p_{1}$ and $p_{2}$ (here we rely on the positive definiteness of $M$ ). By taking two boundary measurements we have thus directly obtained an approximation to the rescaled polarization tensor as well as the location of the inhomogeneity. The above formulas are in the same spirit as those derived in [2] for the identification of a single linear crack. 
Remark 4. If there are more than one inhomogeneity but we know a priori that they all have the same rescaled polarization tensor, then we can easily determine the number of inhomogeneities by one boundary measurement. Simply apply boundary current $\gamma_{0} \nu_{1}$ (so that $u=x_{1}$ ) and let $w=x_{1}$. Then (2.13) gives

$$
\Gamma \approx \epsilon^{2} m M_{11}
$$

where $\Gamma$ is measured. Assuming $\epsilon^{2} M_{11}$ is known, we obtain $m$ directly.

\section{USING FourieR INVERSION}

In the previous section we saw an example of how the formula (2.13) can be used to directly calculate an approximation to the polarization tensor and the location of one inhomogeneity. By choosing more test functions $w$ and possibly taking more boundary measurements, one may in a quite similar way calculate the locations and/or polarization tensors for several inhomogeneities. For a large number of inhomogeneities of different shape this becomes more difficult as the corresponding systems become highly nonlinear (and potentially, ill-posed). In this section we focus on an alternate (linear) method to determine the locations (and possibly, the shapes) of inhomogeneities of a wide range of types. This approach may require quite a number of boundary measurements, but if these are readily available, then the approach is rapid and simple to implement. This approach is based on the original idea of Calderon [7], which was, by way of the low amplitude perturbation formula (2.15), to reduce the reconstruction problem to the calculation of an inverse Fourier Transform.

We rewrite the formula $(2.13)$

$$
\Gamma=\int_{\partial \Omega} u_{\epsilon} \gamma_{0} \frac{\partial w}{\partial \nu} \mathrm{d} \sigma-\int_{\partial \Omega} \gamma_{\epsilon} \frac{\partial u_{\epsilon}}{\partial \nu} w \mathrm{~d} \sigma \approx \epsilon^{2} \sum_{i=1}^{m} M^{(i)} \nabla_{x} u\left(z_{i}\right) \cdot \nabla_{x} w\left(z_{i}\right)
$$

by absorbing the constant $\gamma_{0}\left(\frac{\gamma_{0}}{\gamma_{j}}-1\right)$ into the matrix $M^{(j)}$. For an arbitrary $\eta \in \mathbb{R}^{2}$, we assume we are in possession of the boundary data for the voltage potential, $u_{\epsilon}$, whose corresponding background potential is given by

$$
u=\mathrm{e}^{i\left(\eta+i \eta^{\perp}\right) \cdot x}
$$

(boundary current $\left.=i\left(\eta+i \eta^{\perp}\right) \cdot \nu \mathrm{e}^{i\left(\eta+i \eta^{\perp}\right) \cdot x}\right)$ and we average this boundary data using the test function

$$
w=\mathrm{e}^{i\left(\eta-i \eta^{\perp}\right) \cdot x}
$$

Note that both of these functions are harmonic. Let us now view the measurement (4.17) as a function of $\eta$ :

$$
\left.\left.\Gamma(\eta) \approx \epsilon^{2} \sum_{j=1}^{m} M^{(j)} \nabla\left(\mathrm{e}^{i\left(\eta+i \eta^{\perp}\right) \cdot x}\right)\right|_{x=z_{j}} \cdot \nabla\left(\mathrm{e}^{i\left(\eta-i \eta^{\perp}\right) \cdot x}\right)\right|_{x=z_{j}}
$$

or

$$
\Gamma(\eta) \approx-\epsilon^{2} \sum_{j=1}^{m} M^{(j)}\left(\eta+i \eta^{\perp}\right) \cdot\left(\eta-i \eta^{\perp}\right) \mathrm{e}^{2 i \eta \cdot z_{j}}
$$

Recall that the function

$$
\mathrm{e}^{2 i \eta \cdot z_{j}}
$$

(up to a multiplicative constant) is exactly the Fourier Transform of the Dirac delta

$$
\delta_{-2 z_{j}}
$$


(a point mass located at $-2 z_{j}$ ). Multiplication by powers of $\eta$ in Fourier space corresponds differentiation of the delta functions. The function $\Gamma(\eta)$ is therefore the Fourier Transform of a linear combination of derivatives of point masses, or

$$
\check{\Gamma}(x) \approx \epsilon^{2} \sum_{j=1}^{m} L_{j} \delta_{-2 z_{j}},
$$

where $L_{j}$ is a second order constant coefficient, differential operator whose coefficients depend on the polarization tensor $M^{(j)}$, and $\check{\Gamma}(x)$ represent the inverse Fourier Transform of $\Gamma(\eta)$. The method of reconstruction we propose here consists in sampling the values of $\Gamma(\eta)$ at some discrete set of points and then calculating the corresponding discrete inverse Fourier Transform. After a rescaling (by -1/2) the support of this inverse Fourier Transform yields the location of the inhomogeneities. Once the locations are known we may calculate the polarization tensors by solving an appropriate linear system arising from (2.13).

Similarly, when we have $m$ inhomogeneities in the form of thin strips

$$
\Gamma(\eta) \approx \epsilon \sum_{j=1}^{m} \int_{\sigma_{0}^{j}} A^{(j)}(x)\left(\eta+i \eta^{\perp}\right) \cdot\left(\eta-i \eta^{\perp}\right) \mathrm{e}^{i 2 \eta \cdot x} \mathrm{~d} s_{x},
$$

where $\gamma_{0}$ as well as $h^{(j)}(x)$ have been absorbed into $A^{(j)}(x)$. This is the Fourier Transform of a linear combination of derivatives of distributions with support on the curves $-2 \sigma_{0}^{j}$, or

$$
\check{\Gamma}(x) \approx \epsilon \sum_{j=1}^{m} L_{j} D_{\sigma_{0}^{j}}
$$

where $\check{\Gamma}(x)$ denotes the inverse Fourier Transform of $\Gamma(\eta)$, the the $L_{j}$ are second order constant coefficient differential operators, and $D_{\sigma_{0}^{j}}$ are distributions supported on $-2 \sigma_{0}^{j}$. In this case we also propose to calculate a discrete inverse Fourier Transform of $\Gamma(\eta)$ to approximate the locations and shapes of the curves $\sigma_{0}^{j}$.

Calderon's analysis ( $c f$. [7]) showed that the inverse Fourier Transform of $\Gamma(\eta)$ yielded a good approximation to small amplitude perturbations in the conductivity (around a constant). The calculations above shows that it also yields a good approximation to (large amplitude but) small volume fraction perturbations of the conductivity. Finally we note that it is possible to construct exponential solutions, with properties similar to those above, for use in more complicated situations, for instance in connection with the Maxwell equations (see [17]).

\section{Numerical Results}

In the last section we saw that the boundary measurement

$$
\Gamma(\eta)=\int_{\partial \Omega} u_{\epsilon} \gamma_{0} \frac{\partial w}{\partial \nu} \mathrm{d} \sigma-\int_{\partial \Omega} \gamma_{\epsilon} \frac{\partial u_{\epsilon}}{\partial \nu} w \mathrm{~d} \sigma
$$

corresponding to background potential

and test function

$$
\begin{aligned}
& u=\mathrm{e}^{i\left(\eta+i \eta^{\perp}\right) \cdot x}, \\
& w=\mathrm{e}^{i\left(\eta-i \eta^{\perp}\right) \cdot x}
\end{aligned}
$$

is approximately (up to rescalings) the Fourier Transform of a distribution with support at the centers of the inhomogeneities. We now consider the computational aspects of performing a discrete inverse Fourier Transform in order to identify the location of the inhomogeneities. Since the accuracy of the approximations $(1.4,1.10)$ (and thus the accuracy of the approximations $(2.13,2.14)$ ) has already been carefully documented the most important computational issue that remains is the conditioning of the discrete inverse Fourier Transform, when applied to data that arise from distributions that are supported at a finite set of points or on a finite set of simple 
curves. Again, reflecting the fact that the accuracy of the approximations (2.13) and (2.14) are not at issue, we shall use these two formulas to generate the data for $\Gamma(\eta)$ (as opposed to computing it from approximate solutions to the elliptic boundary value problems). We do want to emphasize that before one could contemplate to use this algorithm "in real life" there is of course one important practical issue, which we do not attempt to address, and which needs to be resolved. We are here referring to the issue of how to obtain a sufficient number of real measurements to generate the values of $\Gamma(\eta)$ needed for a satisfactory Fourier Inversion.

To arrive at some idea of the number of data (sampling) points needed for an accurate discrete Fourier inversion we remind the reader of the main assertion of the so-called Shannon's Sampling theorem (cf. [9]): "a function $f$ is completely specified (by a very explicit formula) by the sampled values $\left\{f\left(\frac{n}{2 M}+c_{0}\right)\right\}_{n=-\infty}^{\infty}$ if and only if the support of $\hat{f}$ is contained inside a square of side $2 M$ ". For our application this suggests two things: (1) if the inhomogeneities are contained inside a square of side $2 M$, then we need to sample $\Gamma(\eta)$ at a uniform, infinite, rectangular grid of meshsize $\frac{1}{2 M}$ to obtain an accurate reconstruction, (2) if we only sample the points in this grid for which the absolute values of the coordinates are less than $K$, then the resulting discrete inverse Fourier Transform will recover the location of the inhomogeneities with a resolution of order $\delta=\frac{1}{2 K}$. In summary: we need (conservatively) of the order $(2 M / \delta)^{2}$ sampled values of $\Gamma(\eta)$ to reconstruct, with resolution $\delta$, a collection of inhomogeneities that lie inside a square of side $2 M$.

It may be useful to compare these measurement counts to those related to another method (a linear "linear sampling" method) which has recently been proposed as a tool for the reconstruction of collections of small inhomogeneities (see [6]). For the accurate reconstruction of the location of $m$ inhomogeneities this method requires knowledge of the subspace spanned by the first $2 m$ eigenvectors of the incremental Dirichlet to Neumann data operator. For moderate size $m$ it is reasonable to assume that we are able to approximate this subspace (so as to determine the location of the inhomogeneities with a resolution of oder $\delta$ ) by $C_{\delta} m$ measurements (using a "power-like" method) where $C_{\delta}$ depends on $\delta$ and (the gaps in) the spectrum of the incremental Dirichlet to Neumann data operator. For a relatively small number of inhomogeneities $\left(m \ll(2 M / \delta)^{2} / C_{\delta}\right)$ the "linear sampling" method would therefore require fewer measurements than the Fourier Inversion based approach. For relatively large number of inhomogeneities the count should be in favor of the Fourier Inversion based approach. We note that the largest possible number of inhomogeneities that are well separated (by $\delta$ ) is $a$ priori bounded by $(2 M / \delta)^{2}$. A more precise comparison of measurement counts would require a careful analysis of the actual size of $C_{\delta}$ and the range of validity of the bound $C_{\delta} \mathrm{m}$. We also note that the present crude comparison may substantially overestimate the number of measurements needed for the Fourier Inversion based approach in the case of only a few inhomogeneities. Some of the following numerical examples clearly indicate that fewer measurents are sufficient to reconstruct the location of a very small number of inhomogeneities.

As our first example, we take the domain $\Omega$ to be the square $[-10,10] \times[-10,10]$ and we insert five inhomogeneities in the shape of balls, with the $j$-th ball positioned at the point $(j, j)$. In the case of balls the polarization tensor is just a multiple of the identity, $M^{(j)}=d_{j} I$, where $d_{j}$ depends on the aspect ratio $\gamma_{0} / \gamma_{j}$ and the relative size of the ball [8]. Here we take each $d_{j}$ to be 10 and $\epsilon=0.1$. We sample our approximation of $\Gamma(\eta)$ on the square $[-3,3] \times[-3,3]$ with a uniform $30 \times 30$ grid. We are thus following the recipe from above, with $M=2.5$ and $K=3$. We should expect a recovery of all the locations of the inhomogeneities, with a resolution $\delta=1 / 6$. The discrete inverse Fourier Transform yields the grey-level (intensity) plot shown in Figure 1.

Note, though, that the reconstruction in Figure 1 corresponds to sampling $\Gamma(\eta)$ at 900 points. Next we sample the same function on $[-2.5,2.5] \times[-2.5,2.5]$ with a uniform $20 \times 20$ grid, thus having a total of 400 sample points. In Figure 2 we see that the five balls are still visible. If the domain has only one inhomogeneity, then we can find its location by sampling far fewer values of $\Gamma$. The reconstruction shown in Figure 3 corresponds to a single inhomogeneity at the point $(1,1)$. We have sampled the values of $\Gamma$ on the square $[-1.5,1.5] \times[-1.5,1.5]$ using a uniform $4 \times 4$ grid.

In order to simulate errors in the boundary measurements, as well as the error inherent in the approximations $(2.13,2.14)$ we add on the order of $10 \%$ of random noise to the values of $\Gamma$ in the examples with five inhomogeneities. We see from Figures 4 and 5 that the reconstruction is quite stable. 


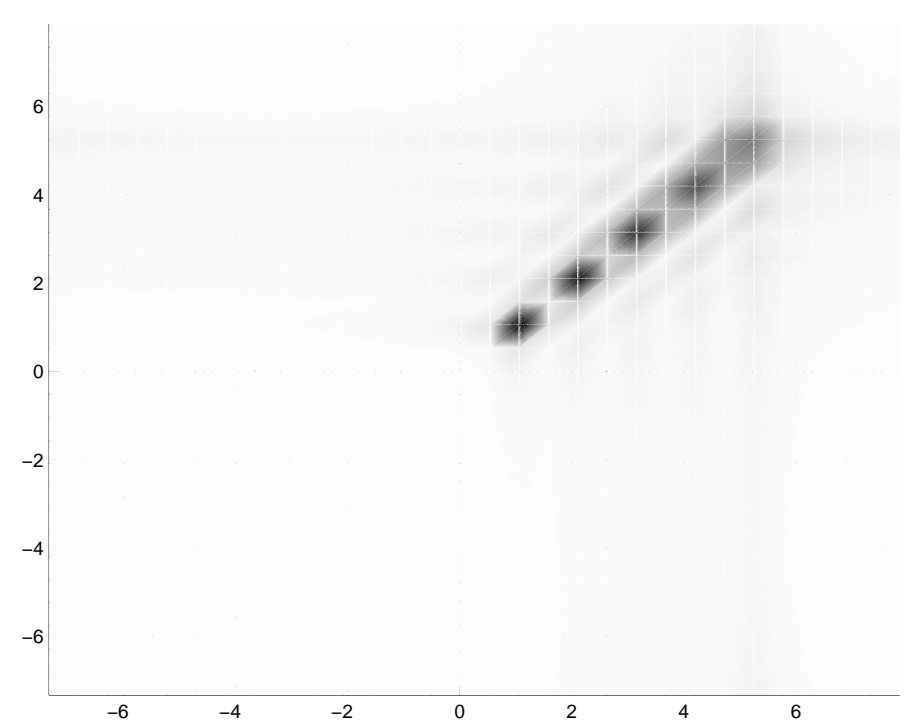

FiguRE 1. Five inhomogeneities $-30 \times 30$ sample points.

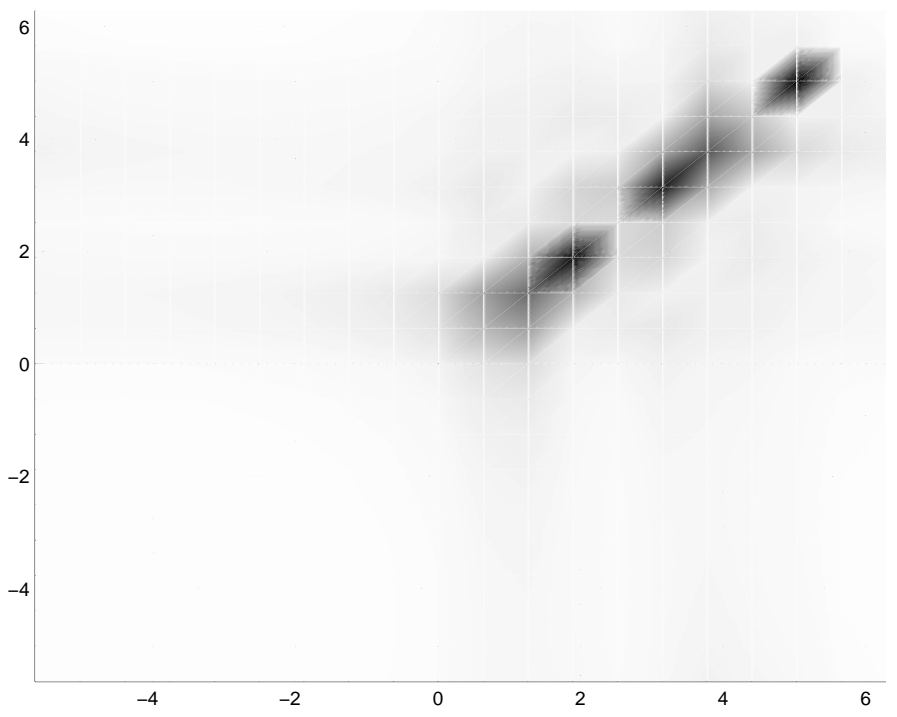

Figure 2. Five inhomogeneities $-20 \times 20$ sample points.

For our next example we take the case in which there are 50 equidistant balls (of same size and aspect ratio as before) lying on the diagonal between $(-5,5)$ and $(5,5)$. We sample the approximation to $\Gamma(\eta)$ (with $10 \%$ noise added) on the square $[-10,10] \times[-10,10]$ using a uniform $128 \times 128$ grid. From Figure 6 see that the placement of the 50 balls is clearly defined, but on the other hand we can not identify individual balls. We note that, due to the closeness of the balls, it is not clear how good an approximation to $\Gamma$ the expression (2.13) provides. We also note that "background" noise is more visible here than in any of the previous plots: this is entirely to be expected, since the fairly high number of balls leads to much larger values for the "averaged data" and thus to much larger absolute noise. The "maximal intensity" near the location of the balls is also 


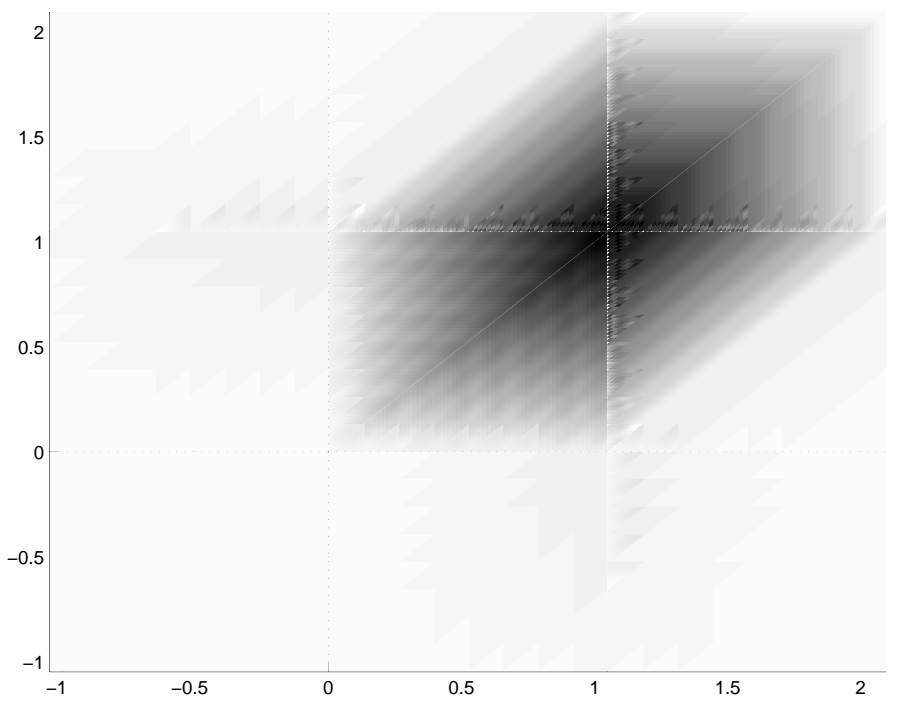

FiguRE 3. One inhomogeneity $-4 \times 4$ sample points.

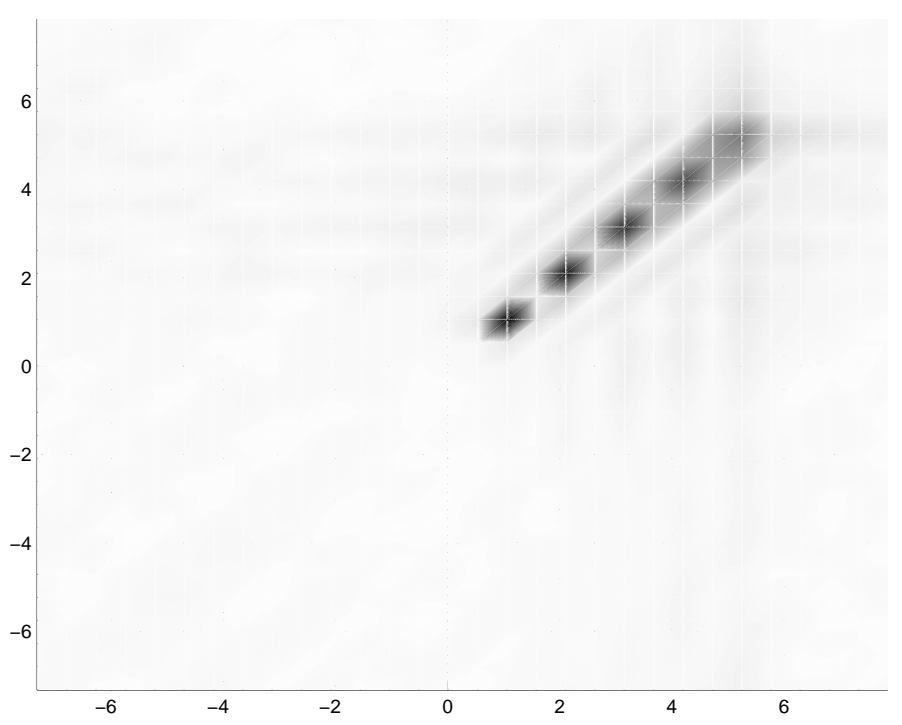

Figure 4. Five inhomogeneities $-30 \times 30$ sample points with $10 \%$ noise.

significantly larger than before; this may be a little hard to appreciate on a grey-level plot, it is much easier to see on the corresponding surface plot, shown in Figure 7.

In our last example we take an inhomogeneity in the shape of a thin strip, i.e. we use the approximation to $\Gamma(\eta)$ arising from $(2.14)$. The strip is situated about the interval $[0,1]$ on the $x$-axis. We choose the background conductivity to be 1 and the conductivity on the strip to be 10 , and take $\epsilon=0.1$. We sample the approximation to $\Gamma(\eta)$ with $(10 \%$ noise added $)$ on the square $[-3,3] \times[-3,3]$ using a uniform $10 \times 10$ grid. The position of the strip is clearly visible in Figure 8.

The numerical experiments we have presented here correspond to but one of many possible choices for the background voltages and the test functions in the formulae (2.13) and (2.14). We are currently exploring other 


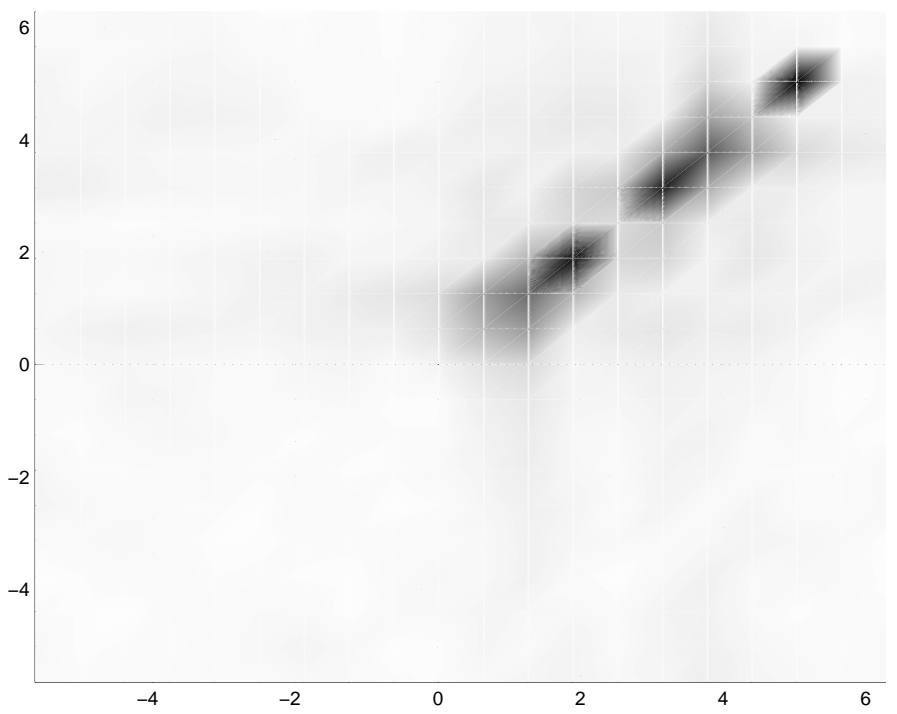

FiguRE 5 . Five inhomogeneities $-20 \times 20$ sample points with $10 \%$ noise.

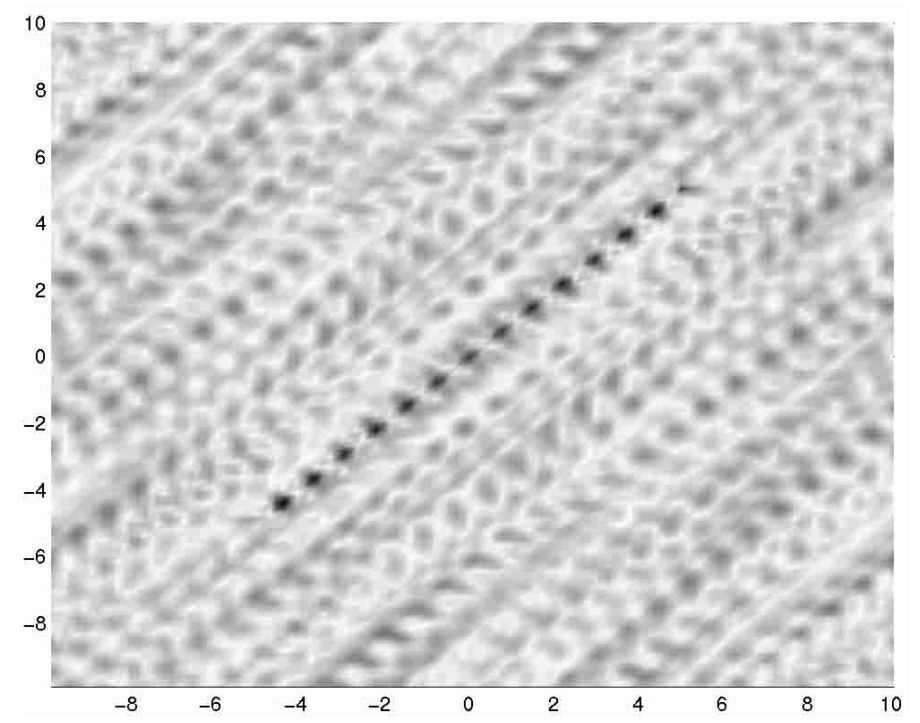

FIGURE 6. Fifty inhomogeneities $-128 \times 128$ sample points with $10 \%$ noise.

choices, some of which are undoubtedly more practical, as far as the required number of measurements are concerned. We are convinced that the use of approximate formulae such as (2.13) and (2.14) represents a very promising approach to the reconstruction problem, in particular when one considers reconstructions based on the use of electric as well as magnetic fields. This is the main reason why we devote the next section to the (formal) derivation of a formula analogous to (2.13) for solutions to the Maxwell equations. 


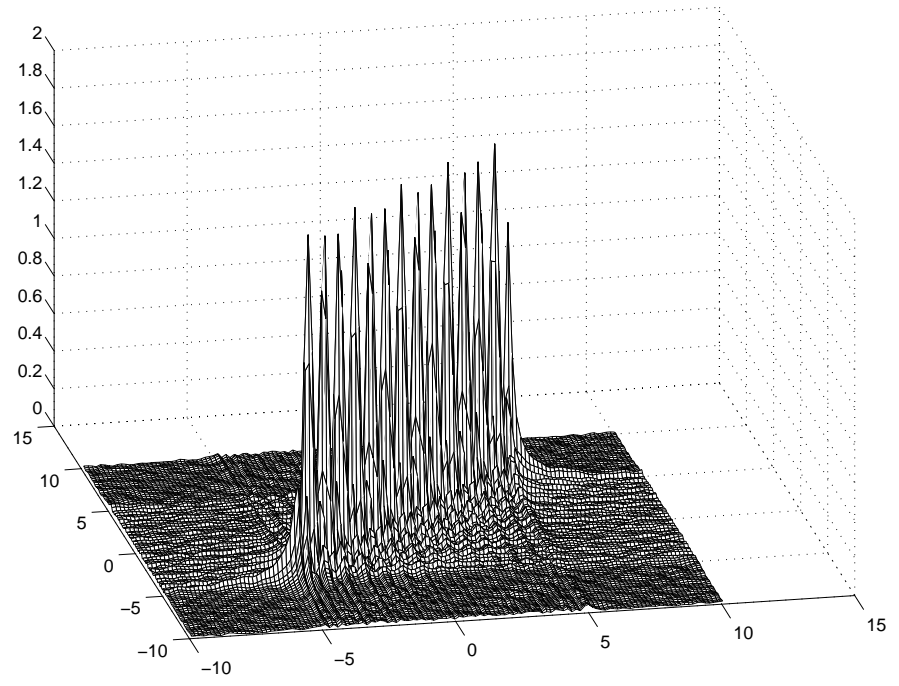

Figure 7 . Fifty inhomogeneities $-128 \times 128$ sample points with $10 \%$ noise.

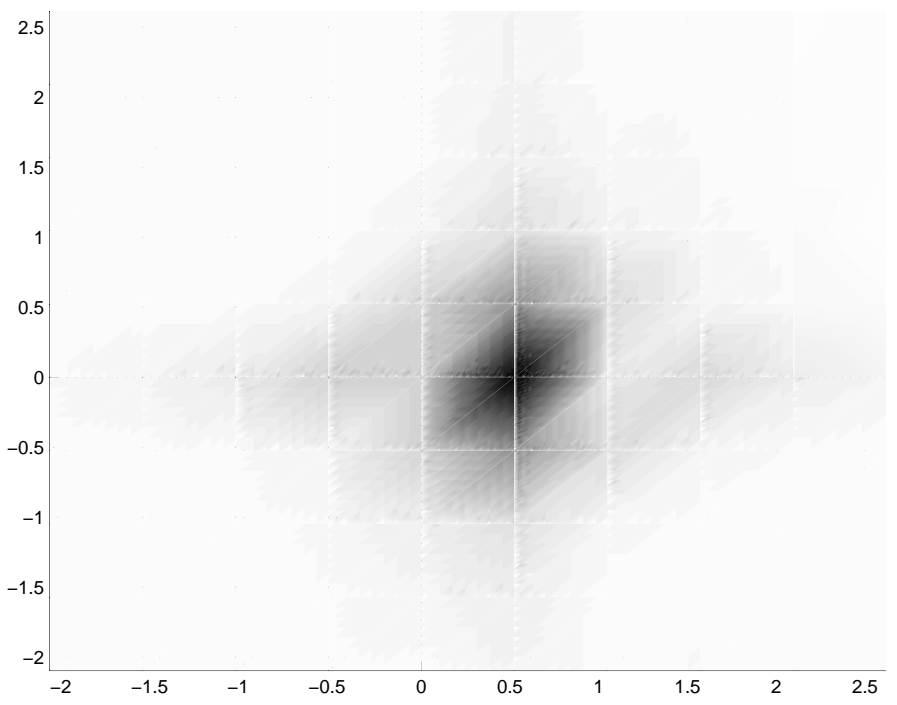

Figure 8 . Strip $-10 \times 10$ sample points with $10 \%$ noise.

\section{IDENTIFICATION OF SMALL INHOMOGENEITIES USING ELECTROMAGNETIC FIELDS}

In this section we establish the basis for the extension of our reconstruction approach to take into account all electromagnetic fields. To be more precise: we give a formal derivation of an identity similar to (2.13) for solutions to the 3-d Maxwell equations (the identity (6.32)). This formal derivation provides an extremely intuitive and accessible alternative to the rigorous, at times quite technical, derivation found in [1]. We strongly feel that the additional insight provided by this formal derivation will allow the discovery of similar identities in other contexts. 
Let $\Omega$ be a bounded subset of $\mathbb{R}^{3}$, with smooth boundary $\partial \Omega$. Let $\nu$ denote the outward unit normal to $\partial \Omega$, and let $\left(k_{1}, k_{2}, k_{3}\right)$ be an orthonormal basis of $\mathbb{R}^{3}$. For fixed frequency $\omega>0,\left(E_{\epsilon}, H_{\epsilon}\right)$ denote time-harmonic electromagnetic fields. These time-harmonic fields satisfy the following form of the Maxwell equations

$$
\begin{aligned}
& \nabla \times E_{\epsilon}=i \omega \mu_{\epsilon} H_{\varepsilon} \quad \text { in } \Omega, \\
& \nabla \times H_{\epsilon}=-i \omega q_{\epsilon} E_{\epsilon} \text { in } \Omega \text {, } \\
& E_{\epsilon} \times \nu=f \quad \text { on } \partial \Omega \text {. }
\end{aligned}
$$

Here $f$ is a tangential field on the boundary $\partial \Omega$ that furthermore belongs to the Sobolev $\operatorname{space} H_{\text {div }}^{-1 / 2}(\partial \Omega)=$ $\left\{g \in H^{-1 / 2}(\partial \Omega)^{3}, g \cdot \nu=0\right.$ on $\left.\partial \Omega, \operatorname{div}_{\partial \Omega}(g) \in H^{-1 / 2}(\partial \Omega)\right\}$. The magnetic permeability $\mu_{\varepsilon}$ and the electric permittivity $q_{\varepsilon}$ differ from the (background) values $\mu_{0}$ and $q_{0}$ inside the small inhomogeneity $B_{\epsilon}=\epsilon B+z$. In other words

$$
\mu_{\epsilon}= \begin{cases}\mu_{0} & \text { in } \Omega \backslash \overline{B_{\epsilon}}, \\ \mu_{1} & \text { in } B_{\epsilon},\end{cases}
$$

and

$$
q_{\epsilon}= \begin{cases}q_{0} & \text { in } \Omega \backslash \overline{B_{\epsilon}} \\ q_{1} & \text { in } B_{\epsilon} .\end{cases}
$$

$B$ is a bounded smooth domain, containing the origin; $\mu_{j}$ and $q_{j}$ are constants with $\mu_{j}>0$, Re $q_{j}>0$, and $\operatorname{Im} q_{j} \geq 0$. For simplicity we only consider one inhomogeneity $B_{\epsilon}$. The general case (with a finite number of well separated inhomogeneities) does not lead to any new difficulties. We assume that $\omega$ is not an eigenvalue for the problem (6.18), and so by assumption there exists a unique solution $\left(E_{\epsilon}, H_{\epsilon}\right) \in[H(\operatorname{curl}, \Omega)]^{2}=\{F \in$ $\left.L^{2}(\Omega)^{3}, \nabla \times F \in L^{2}(\Omega)^{3}\right\}^{2}$. From (6.18) we have that

$$
\nabla \cdot\left(q_{\epsilon} E_{\epsilon}\right)=\nabla \cdot\left(\mu_{\epsilon} H_{\epsilon}\right)=0 \quad \text { in } \Omega
$$

From [15] and [13] we know that the boundary map given by

$$
\Lambda: E_{\epsilon} \times\left.\nu\right|_{\partial \Omega} \mapsto H_{\epsilon} \times\left.\nu\right|_{\partial \Omega}
$$

where $E_{\epsilon}, H_{\epsilon}$ satisfy (6.18) determines the functions $\mu_{\varepsilon}$ and $q_{\varepsilon}$, in the case when these are sufficiently smooth. The goal in the piecewise constant context, considered here, is to construct an efficient method for determining the location of $B_{\epsilon}$, and certain aspects of $B$ from only one pair $\left(E_{\epsilon} \times \nu, H_{\epsilon} \times \nu\right)$.

Let $v$ be a smooth solution to

$$
\nabla \times(\nabla \times v)-\omega^{2} \mu_{0} q_{0} v=0
$$

From the Maxwell equations (6.18) we obtain by integration by parts

$$
\begin{aligned}
& \int_{\partial \Omega}\left(\nabla \times E_{\epsilon}\right) \times \nu \cdot v \mathrm{~d} \sigma-\int_{\partial \Omega}(\nabla \times v) \times \nu \cdot E_{\epsilon} \mathrm{d} \sigma=\omega^{2} \mu_{0}\left(q_{0}-q_{1}\right) \int_{B_{\epsilon}} E_{\epsilon} \cdot v \mathrm{~d} x \\
& \quad+\left(\frac{\mu_{0}}{\mu_{1}}-1\right) \int_{B_{\epsilon}} \nabla \times E_{\epsilon} \cdot \nabla \times v \mathrm{~d} x .
\end{aligned}
$$


If we replace $v$ and $\nabla \times v$ by $v(z)$ and $\nabla \times v(z)$ in $B_{\epsilon}$ we formally obtain

$$
\begin{aligned}
\int_{\partial \Omega}(\nabla & \left.\times E_{\epsilon}\right) \times \nu \cdot v \mathrm{~d} \sigma-\int_{\partial \Omega}(\nabla \times v) \times \nu \cdot E_{\epsilon} \mathrm{d} \sigma=\omega^{2} \mu_{0}\left(q_{0}-q_{1}\right)\left(\int_{B_{\epsilon}} E_{\epsilon} \mathrm{d} x\right) \cdot v(z) \\
& +\left(\frac{\mu_{0}}{\mu_{1}}-1\right)\left(\int_{B_{\epsilon}} \nabla \times E_{\epsilon} \mathrm{d} x\right) \cdot \nabla \times v(z)+o\left(\epsilon^{3}\right) .
\end{aligned}
$$

We note that it is quite easy, systematically to create vector valued functions $v$, that satisfy (6.20). For example, let $\xi \in \mathbb{R}^{3}$ be such that $\xi \cdot \xi=\omega^{2} \mu_{0} q_{0}$, let $k$ be an arbitrary vector in $\mathbb{R}^{3}$, and define

$$
v_{\xi, k}(x)=\mathrm{e}^{i \xi \cdot x} k+\frac{1}{\omega^{2} \mu_{0} q_{0}} \nabla \nabla \cdot\left(\mathrm{e}^{i \xi \cdot x} k\right)=\mathrm{e}^{i \xi \cdot x}\left(k-\frac{k \cdot \xi}{\omega^{2} \mu_{0} q_{0}} \xi\right)
$$

In order to derive a formula that is similar to (2.13) we first derive an asymptotic expansion for $E_{\epsilon}$ inside $B_{\epsilon}$, which we then insert into (6.22).

\subsection{The asymptotic expansion for $E_{\epsilon}$}

Following a common practice in multiscale expansions we introduce the local variable $y=\frac{x-z}{\epsilon}$, where $z$ is the "center" of $B_{\epsilon}$. Let $\left(E_{0}, H_{0}\right)$ be the solution to the following (background) Maxwell Equations

$$
\begin{array}{ll}
\nabla \times E_{0}=i \omega \mu_{0} H_{0} & \text { in } \quad \Omega, \\
\nabla \times H_{0}=-i \omega q_{0} E_{0} & \text { in } \quad \Omega, \\
E_{0} \times \nu=f & \text { on } \partial \Omega .
\end{array}
$$

We expect that $E_{\epsilon}(x)$ will differ appreciably from $E_{0}(x)$ for $x$ near $z$, but that it will differ little from $E_{0}(x)$ for $x$ far from $z$. Therefore, in the spirit of matched asymptotic expansions, we shall represent $E_{\epsilon}(x)$ by two different expansions, an inner expansion for $x$ near $z$, and an outer expansion for $x$ far from $z$. The outer expansion must begin with $E_{0}$, so we write

$$
E_{\epsilon}(x)=E_{0}(x)+\epsilon^{\alpha_{1}} E_{1}(x)+\epsilon^{\alpha_{2}} E_{2}(x)+\ldots, \quad \text { for }|x-z| \gg O(\epsilon),
$$

where $0<\alpha_{1}<\alpha_{2}<\ldots$, and $E_{1}, E_{2}, \ldots$ are to be found. We write the inner expansion as

$$
E_{\epsilon}(z+\epsilon y)=e_{\epsilon}(y)=e_{0}(y)+\epsilon e_{1}(y)+\epsilon^{2} e_{2}(y)+\ldots \text { for }|y|=O(1)
$$

where $e_{0}, e_{1}, \ldots$ are to be found. The magnetic field $H_{\epsilon}$ has similar expansions. The inner and outer expansions must be asymptotically equal in some "overlap" domain within which the stretched variable $y$ is large and $x-z$ is small. In this domain the matching condition is

$$
E_{0}(x)+\epsilon^{\alpha_{1}} E_{1}(x)+\epsilon^{\alpha_{2}} E_{2}(x)+\ldots \sim e_{0}(y)+\epsilon e_{1}(y)+\epsilon^{2} e_{2}(y)+\ldots
$$

From the terms of order $\epsilon^{0}$, we obtain the first matching condition

$$
e_{0}(y) \rightarrow E_{0}(z) \quad \text { as }|y| \rightarrow+\infty
$$

If we substitute $(6.26)$ into $(6.18,6.19)$ and formally equate coefficients of $\epsilon^{-1}$ we get

$$
\nabla_{y} \times e_{0}=0, \nabla_{y} \cdot\left(q e_{0}\right)=0 \text { in } \mathbb{R}^{3},
$$


where

$$
q= \begin{cases}q_{0} & \text { in } \mathbb{R}^{3} \backslash \bar{B} \\ q_{1} & \text { in } B\end{cases}
$$

Therefore,

$$
e_{0}(y)=\sum_{i=1}^{3}\left(E_{0}(z) \cdot k_{i}\right) \nabla_{y} \varphi_{i}(y)
$$

where $\varphi_{i}$ is the unique solution to the following problem

$$
\begin{aligned}
& \nabla_{y} \cdot\left(q(y) \nabla_{y} \varphi_{i}\right)=0 \quad \text { in } \mathbb{R}^{3}, \\
& \varphi_{i}(y)-y_{i} \rightarrow 0, \quad \text { as }|y| \rightarrow+\infty .
\end{aligned}
$$

The asymptotic behavior of $\nabla_{y} \varphi_{i}(y)$ as $|y| \rightarrow+\infty$ (see [10]) is

$$
\nabla_{y} \varphi_{i}(y)=k_{i}+\frac{C}{|y|^{3}} y+o\left(\frac{1}{|y|^{2}}\right)
$$

where $C$ is a constant that depends on $q_{0}, q_{1}$, and $B$. We note that the coefficients $q_{j}$ are complex, but the fact that $\operatorname{Re} q_{j}>0$ is sufficient to guarantee the uniqueness and the decay mentioned above.

Exactly as for the electric field, the first order term in the asymptotic expansion of $\frac{1}{i \omega \mu_{1}} \nabla \times E_{\epsilon}=H_{\epsilon}$ inside $B_{\epsilon}$ is given by

$$
h_{0}(y)=\sum_{i=1}^{3}\left(H_{0}(z) \cdot k_{i}\right) \nabla_{y} \psi_{i}(y)
$$

where $\psi_{i}$ is the unique solution to the following problem

$$
\begin{aligned}
& \nabla_{y} \cdot\left(\mu(y) \nabla_{y} \psi_{i}\right)=0 \quad \text { in } \mathbb{R}^{3}, \\
& \psi_{i}(y)-y_{i} \rightarrow 0, \quad \text { as }|y| \rightarrow+\infty,
\end{aligned}
$$

with

$$
\mu= \begin{cases}\mu_{0} & \text { in } \mathbb{R}^{3} \backslash \bar{B}, \\ \mu_{1} & \text { in } B .\end{cases}
$$

Based on Remark 1.7 we immediately get that

$$
\frac{q_{1}}{q_{0}} \int_{B} \frac{\partial}{\partial y_{j}} \varphi_{l}(y) \mathrm{d} y=|B| \delta_{j l}-\int_{\partial B} y_{j} \frac{\partial \phi_{l}^{+}}{\partial \nu} \mathrm{d} \sigma_{y}=M_{j l}\left(\frac{q_{0}}{q_{1}}\right),
$$

where the tensor $M\left(\frac{q_{0}}{q_{1}}\right)$ has been appropriately extended to complex aspect ratios. Similarly we get

$$
\frac{\mu_{1}}{\mu_{0}} \int_{B} \frac{\partial}{\partial y_{j}} \psi_{l}(y) \mathrm{d} y=M_{j l}\left(\frac{\mu_{0}}{\mu_{1}}\right)
$$




\subsection{The asymptotic formula for the measured data}

By combining $(6.22)$ and the (inner) asymptotic expressions $(6.28,6.30)$ we arrive at the following formula.

$$
\begin{aligned}
\int_{\partial \Omega}\left(\nabla \times E_{\epsilon}\right) \times \nu \cdot v \mathrm{~d} \sigma-\int_{\partial \Omega}(\nabla \times v) \times \nu \cdot \nu \times\left(E_{\epsilon} \times \nu\right) \mathrm{d} \sigma \\
=\epsilon^{3} \omega^{2} \mu_{0}\left(q_{0}-q_{1}\right) \sum_{i=1}^{3}\left(E_{0}(z) \cdot k_{i}\right)\left(\int_{B} \nabla_{y} \varphi_{i}(y) \mathrm{d} y\right) \cdot v(z) \\
\quad+\left(\frac{\mu_{0}}{\mu_{1}}-1\right) i \omega \mu_{1} \sum_{i=1}^{3}\left(H_{0}(z) \cdot k_{i}\right)\left(\int_{B} \nabla_{y} \psi_{i}(y) \mathrm{d} y\right) \cdot \nabla \times v(z) \\
=\epsilon^{3}\left[\omega^{2} \mu_{0} q_{0}\left(\frac{q_{0}}{q_{1}}-1\right) M\left(\frac{q_{0}}{q_{1}}\right) E_{0}(z) \cdot v(z)\right. \\
\left.\quad+i \omega \mu_{0}\left(\frac{\mu_{0}}{\mu_{1}}-1\right) M\left(\frac{\mu_{0}}{\mu_{1}}\right) H_{0}(z) \cdot \nabla \times v(z)\right]+o\left(\epsilon^{3}\right) .
\end{aligned}
$$

Here $\left(E_{\epsilon}, H_{\epsilon}\right)$ is the unique solution to $(6.18)$ and $\left(E_{0}, H_{0}\right)$ is the unique solution to the unperturbed problem (6.24). The functions $\varphi_{i}$ and $\psi_{i}$ are defined by (6.29) and (6.31). To proceed from (6.22) to (6.32) we have also used that $\nu \times\left(E_{\epsilon} \times \nu\right)$ is the projection of $E_{\epsilon}$ onto the tangent plane of $\partial \Omega$. For a rigorous derivation of this formula (and other asymptotic representations) we refer the reader to [1]. We may now insert the functions $v_{\xi, k}$ (or other solutions to (6.20) into $(6.32)$ ) to arrive at reconstruction algorithms related to those described in Sections 3-5.

Remark 5. Note that if the inhomogeneity $\epsilon B+z$ (and the domain $\Omega$ ) is cylindrical (in the $x_{3}$ direction) then we have $\psi_{3}=\varphi_{3}=y_{3}$. Furthermore, the (correctly interpreted) functions $\psi_{1}, \psi_{2}, \varphi_{1}$ and $\varphi_{2}$ depend only on the variables $\left(y_{1}, y_{2}\right)$, and thus the polarization tensor $\epsilon^{3} M(c)$ gets replaced by

$$
\epsilon^{2} M(c)=\epsilon^{2}\left[\begin{array}{ll}
\left\{M_{\alpha, \beta}(c)\right\}_{\alpha, \beta=1}^{2} & 0 \\
0 & c^{-1}\left|B^{\prime}\right|
\end{array}\right]
$$

If we now consider the TE (transverse electric) case - that is the case of an electric field $E=\left(0,0, E\left(x_{1}, x_{2}\right)\right)$ with corresponding magnetic field $H=\frac{1}{i \omega \mu}\left(\frac{\partial}{\partial x_{2}} E,-\frac{\partial}{\partial x_{1}} E, 0\right)-$ and take $v=\left(0,0, v\left(x_{1}, x_{2}\right)\right)\left(\right.$ with $\left.\triangle v+\omega^{2} \mu_{0} q_{0} v=0\right)$ then the analogue of $(6.32)$ reads

$$
\begin{gathered}
\int_{\partial \Omega^{\prime}} \frac{\partial E_{\epsilon}}{\partial \nu} v \mathrm{~d} \sigma-\int_{\partial \Omega^{\prime}} \frac{\partial v}{\partial \nu} E_{\epsilon} \mathrm{d} \sigma=\epsilon^{2}\left[\omega^{2} \mu_{0}\left(q_{0}-q_{1}\right)\left|B^{\prime}\right| E_{0}\left(z^{\prime}\right) v\left(z^{\prime}\right)\right. \\
\left.+\left(\frac{\mu_{0}}{\mu_{1}}-1\right) M\left(\frac{\mu_{0}}{\mu_{1}}\right) R^{T} \nabla E_{0}\left(z^{\prime}\right) \cdot R^{T} \nabla v\left(z^{\prime}\right)\right]+o\left(\epsilon^{2}\right) \\
=\epsilon^{2}\left[\omega^{2} \mu_{0}\left(q_{0}-q_{1}\right)\left|B^{\prime}\right| E_{0}\left(z^{\prime}\right) v\left(z^{\prime}\right)\right. \\
\left.+\left(\frac{\mu_{0}}{\mu_{1}}-1\right) \frac{\mu_{1}}{\mu_{0}} M\left(\frac{\mu_{1}}{\mu_{0}}\right) \nabla E_{0}\left(z^{\prime}\right) \cdot \nabla v\left(z^{\prime}\right)\right]+o\left(\epsilon^{2}\right)
\end{gathered}
$$


where $B^{\prime}, \Omega^{\prime}$ and $z^{\prime}$ denote the two dimensional base domains (or point) of $B, \Omega$ and $z$, respectively. In the last identity we used the second observation from Remark 1.7. The formula (6.33) is closely related to the boundary representations, rigorously derived in [16]. Indeed, the formula (6.33) may obtained by appropriate integration of a slight variation of the representation given in Theorem 1 of [16].

This research was partially supported by the National Science Foundation under grants DMS-9704575 (MSV) and INT-9815798 (HA and SM).

\section{REFERENCES}

[1] H. Ammari, M.S. Vogelius and D. Volkov, Asymptotic formulas for perturbations in the electromagnetic fields due to the presence of inhomogeneities of small diameter II. The full Maxwell Equations. J. Math. Pures Appl. 80 (2001) 769-814.

[2] S. Andrieux and A. Ben Abda, Identification of planar cracks by complete overdetermined data: Inversion formulae. Inverse Problems 12 (1996) 553-563.

[3] S. Andrieux, A. Ben Abda and M. Jaoua, On the inverse emerging plane crack problem. Math. Meth. Appl. Sci. 21 (1998) 895-907.

[4] H.D. Bui, A. Constantinescu and H. Maigre, Diffraction acoustique inverse de fissure plane : solution explicite pour un solide borné. C. R. Acad. Sci. Paris Sér. II 327 (1999) 971-976.

[5] E. Beretta, A. Mukherjee and M. Vogelius, Asymptotic formuli for steady state voltage potentials in the presence of conductivity imperfection of small area. ZAMP $\mathbf{5 2}$ (2001) 543-572.

[6] M. Brühl, M. Hanke and M.S. Vogelius, A direct impedance tomography algorithm for locating small inhomogeneities. Preprint (2001).

[7] A.P. Calderon, On an inverse boundary value problem, in Seminar on Numerical Analysis and its Applications to Continuum Physics. Soc. Brasileira de Matemática, Rio de Janeiro (1980) 65-73.

[8] D.J. Cedio-Fengya, S. Moskow and M.S. Vogelius, Identification of conductivity inperfections of small diameter by boundary measurements. Continuous dependence and computational reconstruction. Inverse Problems 14 (1998) 553-595.

[9] I. Daubechies, Ten Lectures on Wavelets. SIAM, Philadelphia (1992).

[10] G.B. Folland, Introduction to Partial Differential Equations. Princeton University Press, Princeton (1976).

[11] A. Friedman and M. Vogelius, Identification of Small Inhomogeneities of Extreme Conductivity by Boundary Measurements: A Theorem on Continuous Dependence. Arch. Rational Mech. Anal. 105 (1989) 299-326.

[12] S. He and V.G. Romanov, Identification of small flaws in conductors using magnetostatic measurements. Math. Comput. Simul. 50 (1999) 457-471.

[13] M.S. Joshi and S.R. McDowall, Total determination of material parameters from electromagnetic boundary information. Pacific J. Math. (to appear).

[14] K. Miller, Stabilized numerical analytic prolongation with poles. SIAM J. Appl. Math. 18 (1970) 346-363.

[15] P. Ola, L. Païvärinta and E. Somersalo, An inverse boundary value problem in electrodynamics. Duke Math. J. 70 (1993) 617-653.

[16] M.S. Vogelius and D. Volkov, Asymptotic formulas for perturbations in the electromagnetic fields due to the presence of inhomogeneities of small diameter. ESAIM: $M^{2} A N 34$ (2000) 723-748.

[17] D. Volkov, An inverse problem for the time harmonic Maxwell Equations, Ph.D. Thesis. Rutgers University (2001). 